\title{
The Role of Personal and Shared Displays in Scripted Collaborative Learning
}

\author{
Sara Streng \\ Media Informatics, University of Munich, University of Munich, \\ Amalienstr. 17, 80333 Munich, Germany \\ sara.streng@ifi.lmu.de
}

\begin{abstract}
Applying technologies in collaborative learning is an important but difficult task. In this work I will investigate ways of supporting "scripted" collaborative learning with technologies. "Scripted" means that the learning phases, activities and tasks are predefined by educationalists to provide some form of guidance. In particular, I focus on the role of personal and shared displays in different learning phases. Furthermore, I examine how user interfaces can be designed in a way that guides the learner's activities. The proposed contribution is a set of guidelines as well as a software framework that support the creation of scripted collaborative learning applications.
\end{abstract}

Keywords: Collaborative Learning, CSCL Scripts, Shareable User Interfaces.

\section{Introduction}

In many learning scenarios, educators expect a benefit from using computer applications for specific purposes. For example, computers are more efficient in processing or visualizing large chunks of information, they facilitate efficient search and history functions as well as simultaneously editable copies, and much more. There are even learning applications that would not be possible without the support of computers, e.g. ArgueGraph [3].

Despite the advantages of computers, their application in learning environments remains a difficult task to solve. Often usability problems are so profound, that many people still prefer pen and paper. In collaborative learning additional problems arise because traditional desktop computers and laptops are not practicable to be used by multiple people at the same time. Although a number of interesting technologies such as interactive surfaces are available nowadays, they are rarely applied in educational fields.

This research aims at analyzing different technological setups and their applicability in various situations in the area of collaborative learning. This includes the choice of technological devices, the seating arrangement, the design of the user interface as well as the way learners are guided. In the long run, the goal is developing computer applications that are as easy to use as the according pen-and-paper scenario, while simultaneously providing the benefits mentioned above. 


\section{Problem Statement and Research Questions}

Educationalists use collaboration scripts to specify how learners should be guided. This guidance is considered necessary because students rarely engage in effective learning activities spontaneously [5]. A script prescribes a sequence of activities to be carried out by the learners. For example, after reading a text paragraph, one learner summarizes its content. The other person listens and subsequently gives feedback. In the end both learners elaborate on the results and switch roles for the next paragraph [2]. The first research question related to this is: Which guidance functions can be provided by technology support in collaborative learning? My fundamental hypothesis is that well-designed computer programs can be used to guide certain aspects of the learners' activities. For example, in argumentative learning students can be prompted to build argumentation sequences or justify their arguments by designing the user interface in a way that suggests the according activities.

A second important question regards the type of implementation to choose. Many collaboration scripts contain alternating individual and collaborative working phases. Accordingly, technology support for scripted collaboration can be designed in a way such that the interfaces, in particular the displays, correspond to activity types: Individual displays (e.g. laptops, PDAs, tabletPCs) may be used for individual phases, shared displays (interactive surfaces, wall displays) for collaborative phases. To what extent does the usage of display types affect variables connected to learning, such as motivation, the subjective effort for the learners, and of course the learning outcome? In more detail, the following sub-questions will be investigated:

- Which devices and user interfaces are suitable for specific phases of collaborative learning scenarios? Is there a positive effect of the phase-specific choice of technology on the learning outcome? Is there a negative effect caused by the integration of several heterogeneous devices on usability, and how can this effect be counter-balanced?

- How can the integration of personal and shared workspaces be optimized technologically, e.g. by smooth handover or by defining personal workspaces as subspaces of a large shared space (e.g. an interactive table)?

- How does the seating arrangement affect technology-supported collaborative learning? E.g. under which conditions is a loose gathering around a large wall display preferable over a tabletop seating arrangement?

\section{Related Work}

Kobbe et al. 5] proposed a specification of collaboration scripts comprising the educational as well as the computer science perspective. It is the most elaborated approach in this direction and the foundation for any standardization or framework for collaboration scripts.

Specific scripts, which have either been adopted by or at least heavily influenced current projects are ArgueGraph [3, MURDER script 2, Social Script [8], Structured Academic Controversy [4] or Peer Reviewing 7]. 


\section{Approach and Methodology}

Coming from an HCI background it is important for me to get familiar with the educational perspective on collaborative learning. As a foundation I chose the field of collaboration scripts for several reasons. First, as the research field is several decades old, there is a collection of scripts that have proven to be effective. These scripts provide a detailed specification of collaborative learning scenarios including important educational aspects that need to be kept in mind when thinking about suitable user interfaces. Second, the specification proposed by Kobbe et al. [5] structures the domain, which allows for an analysis on an abstract level as well.

The chosen methodology is two-fold. On the one hand, hands-on experience will be gathered. By experimenting with personal and shared displays as well as different guidance strategies in various scenarios, the research questions will be explored. The scenarios are derived from effective collaboration scripts that could be improved by using technologies. The ideas on how technology could be applied are sketched and discussed with educational psychologists. During this process $2 \times 2$ factorial experiment designs are built that cover research questions from both HCI as well as educational psychology. The concepts are then implemented and evaluated in small interdisciplinary projects. In the end, guidelines will be created from the gained experience.

In parallel a software framework will be developed, which supports the implementation of scripted collaborative learning applications. The framework will incorporate structural and technical aspects. The structural aspects include components and mechanisms as proposed by Kobbe et al. [5], as well as strategies for learner guidance. The technical aspects concerns the interaction between heterogeneous devices, e.g. application handovers from personal to shared displays or vice versa. Regarding this issue there is already existing work [1] that can be built on. The framework and the mini-projects are built in a coherent, iterative process. The framework supports the development of the applications, which are built in the mini-projects. In reverse, insights from the practical experience will influence the development of the framework.

\section{Research Conducted and Preliminary Results}

Two projects have already produced preliminary results. The first one is a program that supports two learners in training text comprehension based on the MURDER script [2]. Both learners are provided with laptops. In this case a shareable user interface was not chosen because the two learners have oppositional roles (summarizer and listener). Two user studies have been conducted in a seminar to evaluate the usability as well as effects on variables connected to learning. The results show that subjects who used the program were equally motivated and showed similar effort as the control group who used pen and paper. In a post questionnaire about $75 \%$ of the participants stated that they would prefer the computer application over the pen and paper version. 
In the second project a tabletop application for argumentative learning was developed. The Argue Table guides the learners in substantiating their arguments and building argumentation sequences. A preliminary case study suggests that the quality of argumentation can be improved by means of this guidance [6].

For the realization of these projects, a first version of a framework has already been built. However, there are still many complex issues that need to be considered in a more elaborated version.

\section{Summary and Future Steps}

Collaboration scripts offer a great foundation to study the effects of technology in group learning sessions. Taking advantage of these possibilities, I am empirically exploring the impact of using different technologies in group learning sessions. In particular, two research questions are examined: The role of personal and shared displays as well as learner guidance through careful user interface design.

According to my approach there are two goals I want to pursue. First, practical experience will be gained by experimenting with different technological setups in different scenarios, which will in the end be transformed into guidelines. The second goal is developing a framework that supports the design and implementation of scripted collaborative learning applications.

\section{References}

1. Boring, S.: Interacting in multi-display environments. In: Proceedings of the Doctoral Colloqium at the Ubicomp 2007 (2007)

2. Dansereau, D.F., Collins, K., McDonald, B., Holley, C.D., Garland, J., Diekhoff, G.: Development and evaluation of a learning strategy training program. Journal of Educational Psychology 71(1), 64-73 (1979)

3. Jermann, P., Dillenbourg, P.: Elaborating new arguments through a CSCL script. In: Andriessen, J., Baker, M., Suthers, D. (eds.) Arguing to Learn: Confronting Cognitions in Computer-Supported Collaborative Learning environments, pp. 205226. Kluwer Academic Publishers, Amsterdam (2003)

4. Johnson, D.W., Johnson, R.T.: Constructive conflict in the schools. Journal of Social Issues 50(1), 117-137 (1994)

5. Kobbe, L., Weinberger, A., Dillenbourg, P., Harrer, A., Hmlinen, R., Hkkinen, P., Fischer, F.: Specifying computer-supported collaboration scripts. International Journal of Computer-Supported Collaborative Learning 2(2-3), 211-224 (2007)

6. Stegmann, K., Streng, S., Halbinger, M., Koch, J., Fischer, F., Hussmann, H.: eXtremely Simple Scripting (XSS): A framework to speed up the development of computer-supported collaboration scripts. In: CSCL2009 Community Events Proceedings. ISLS (2009)

7. Trahasch, S.: From peer assessment towards collaborative learning. In: Frontiers of Education, FIE 2004, 34th Annual, October 2004, vol. (2) (2004)

8. Weinberger, A., Fischer, F., Stegmann, K.: Computer-supported collaborative learning in higher education: scripts for argumentative knowledge construction in distributed groups. In: Proceedings of the CSCL 2005, pp. 717-726. International Society of the Learning Sciences (2005) 